\title{
FREQUENCY-DEPENDENT SELECTION
}

\author{
BRYAN CLARKE \\ Department of Zoology, West Mains Road, Edinburgh, 9 \\ and \\ PETER O'DONALD \\ Department of Zoology, University College of North Wales, Bangor
}

\section{INTRODUCTION}

Received 30.x.63

WRIGHT (1948) has set up a mathematical model of a balanced polymorphism in which the selective values of the different genotypes depend upon the frequencies of the genes involved. Lewontin (1958) has suggested a similar model. Maynard Smith (1962) has investigated the maintenance of polymorphism by disruptive selection. Apart from these works, frequency-dependent selection has received little attention.

In this paper we describe some models which may be applicable to balanced polymorphisms involving mimicry (Sheppard, I959) or apostatic selection (Clarke, I962a, I962b). In such polymorphisms the selective values depend upon the frequencies of the phenotypes, rather than upon the frequencies of genes. This dependence is reflected in a negative relation between the selective value of a genotype and the frequency of its phenotype.

\section{GENERAL CONDITIONS FOR EQUILIBRIUM}

If $p$ is the frequency of the gene $A, q$ is the frequency of its allele $\mathrm{A}^{1}$, and $p+q=\mathrm{I}$, then in a population mating at random the frequencies of the genotypes $\mathrm{AA}, \mathrm{AA}^{1}$ and $\mathrm{A}^{1} \mathrm{~A}^{1}$ will be in the ratio $p^{2}: 2 p q: q^{2}$.

If the generations are discrete and the selective values of the three genotypes are respectively $a, b$ and $c$, they will reproduce in the ratio $a p^{2}: 2 b p q: c q^{2}$. In the next generation the frequency of the gene $\mathrm{A}^{1}$ will be

$$
q^{\prime}=\frac{b p q+c q^{2}}{a p^{2}+2 b p q+c q^{2}}
$$

and the change in its frequency

$$
\Delta q=\frac{b p q+c q^{2}}{a p^{2}+2 b p q+c q^{2}}-q=\frac{\left(q-q^{2}\right)[b-a-q(2 b-a-c)]}{a+2 q(b-a)-\frac{q}{q^{2}(2 b-a-c)}} .
$$

When there is a balance of gene frequencies, $\Delta q$ will be zero and the non-trivial equilibrium frequency of $\mathrm{A}^{1}$ will be (Fisher, 1930)

$$
\hat{q}=\frac{b-a}{2 b-a-c}
$$


As Lewontin (1958) pointed out, these formulæ are true whether or not the selective values are constant.

\section{SOME MODELS OF FREQUENCY-DEPENDENT SELECTION}

Four models are described below. The first two illustrate the effects of frequency-dependent selection acting alone, and the second two show the results of a combination of frequency-dependent and frequency-independent selection. The first and third models deal with selection acting upon a pair of alleles whose heterozygote exhibits a phenotype which is clearly distinct from both homozygotes, and which resembles neither of them. This could happen if each of the three possible phenotypes mimicked a separate model, or if the heterozygote provided a unique environment for a particular form of parasite. The second and fourth models deal with the commoner situation, in which one allele is completely dominant to the other.

A later paper (Clarke, in press) will consider the effects of frequencydependent selection upon alleles with incomplete dominance or intermediate heterozygotes.

\section{(i) Frequency-dependent selection when the heterozygotes are distinct}

Suppose that frequency-dependent selection acts alone, and that the selective value of each phenotype is negatively related to its frequency. If the heterozygotes behave as a distinct class, then we have

Genotypes

Frequencies after random mating

Selective values

Frequencies after selection

\section{AA}

$$
a=p^{2}
$$$$
p^{2}\left(1-t p^{2}\right)
$$

$\mathrm{AA}^{1}$

$$
b=\stackrel{2 p q}{1}-2 t p q
$$$$
2 p q(1-2 t p q)
$$

$\mathrm{A}^{1} \mathrm{~A}^{1}$

$$
q^{2}
$$$$
c=\mathrm{I}-t q^{2}
$$$$
q^{2}\left(\mathrm{I}-t q^{2}\right)
$$

where $t$ represents the relation between phenotype-frequency and selective value.

When a mutant gene appears in a population, it will spread if the heterozygote is at a selective advantage to the wildtype homozygote. If the allele $\mathrm{A}^{1}$ newly arises by mutation, $q$ will be extremely small and therefore $b$ will be greater than $a\left(\mathrm{I}-2 t p q>\mathrm{I}-t p^{2}\right)$. The gene will spread. Similarly, if A appears in a population composed entirely of $\mathrm{A}^{1}, b$ will be greater than $c$, and $\mathrm{A}$ will increase in frequency.

If the values of $a, b$ and $c$ given above are substituted in equation (3), the equilibrium gene-frequency is found to be $\hat{q}=0.5$ whatever the value of $t$. At equilibrium $a=\mathrm{I}-t / 4, b=\mathrm{I}-t / 2, c=\mathrm{I}-t / 4$. The heterozygote is thus at a selective disadvantage to both homozygotes.

It can easily be shown that this equilibrium is stable. If $p=\frac{1}{2}+x$ 
and $q=\frac{1}{2}-x$, when $x$ is small the terms in $x^{2}$ and above can be neglected. The value of $x$ in the next generation is then

$$
x^{\prime}=\frac{(8-4 t)}{(8-3 t)} x
$$

Thus $x^{\prime}<x$ as long as $t>0$. The equilibrium is therefore stable.

(ii) Frequency-dependent selection with dominance

If the allele $\mathrm{A}$ is dominant to $\mathrm{A}^{1}$ for characters under selection, we have

\section{Genotypes}

Frequencies after random mating

Selective values

Frequencies after selection
$\mathrm{AA} \quad \mathrm{AA}^{1}$

$$
\begin{array}{ccc}
p^{2} & 2 p q & q^{2} \\
a=\mathrm{I}-t+t q^{2} & b=\mathrm{I}-t+t q^{2} & c=\mathrm{I}-t q^{2} \\
p^{2}\left(\mathrm{I}-t+t q^{2}\right) & 2 p q\left(\mathrm{I}-t+t q^{2}\right) & q^{2}\left(\mathrm{I}-t q^{2}\right) .
\end{array}
$$

Since $b=a$, the allele $\mathrm{A}^{1}$ will spread only very slowly from an initially low frequency. The allele $\mathrm{A}$, on the other hand, will spread more quickly, since when $q$ is large $b$ will be greater than $c$.

Substituting the values of $a, b$ and $c$ in equation (3), we find that $\hat{q}=\sqrt{0.5}=0.7071$ whatever the value of $t$. At equilibrium the heterozygote has a selective value equal to that of the homozygote $\mathrm{AA}$, and the two phenotypes are present in equal numbers. The equilibrium is stable because, if $p=1-\sqrt{0.5}+x$ and $q=\sqrt{0.5}-x$, we find that

$$
x^{\prime}=\left(\frac{\mathrm{I}+0 \cdot 5 t-t \sqrt{2}}{\mathrm{I}-0 \cdot 5^{t}}\right) x
$$

and $x^{\prime}<x$ for any positive value of $t$.

(iii) Combined effects of frequency-dependent and frequency-independent selection when the heterozygotes are distinct

It is very likely that in natural conditions some components of the selective forces will be independent of the frequencies of the phenotypes. When the heterozygote shows a clearly distinct phenotype the situation can be represented as follows:

Genotypes

$\mathrm{AA}$

$\mathrm{AA}^{1}$

$\mathrm{A}^{1} \mathrm{~A}^{1}$

Selective values $a=\mathrm{W}_{1}\left(\mathrm{I}-t p^{2}\right) \quad b=\mathrm{W}_{2}(\mathrm{I}-2 t p q) \quad c=\mathrm{W}_{3}\left(\mathrm{I}-t q^{2}\right)$.

$\mathrm{W}_{1}, \mathrm{~W}_{2}$ and $\mathrm{W}_{3}$ are the frequency-independent components of the selective values of the three genotypes. 
The gene $\mathrm{A}^{1}$ will spread from an initially low frequency if

$$
\mathrm{W}_{2}(\mathrm{I}-2 t q)>\mathrm{W}_{1}(\mathrm{I}-t+2 t q),
$$

and similarly A will spread if

$$
\mathrm{W}_{2}(\mathrm{I}-2 t p)>\mathrm{W}_{3}(\mathrm{I}-t+2 t p) .
$$

Substituting the values of $a, b$ and $c$ in equation (3), we find that $\hat{q}$ is the solution of

$$
\begin{aligned}
& \mathrm{W}_{2}-\mathrm{W}_{1}+t \mathrm{~W}_{1}-\hat{q}\left(2 t \mathrm{~W}_{2}+3 t \mathrm{~W}_{1}+2 \mathrm{~W}_{2}-\mathrm{W}_{1}-\mathrm{W}_{3}\right) \\
& +3 t \hat{q}^{2}\left(2 \mathrm{~W}_{2}+\mathrm{W}_{1}\right)-t \hat{q}^{3}\left(4 \mathrm{~W}_{2}+\mathrm{W}_{1}+\mathrm{W}_{3}\right)=0 .
\end{aligned}
$$

Certain values of $\mathrm{W}_{1}, \mathrm{~W}_{2}, \mathrm{~W}_{3}$ and $t$ will give three solutions between 0 and $\mathrm{I}$. In other words, certain combinations of frequency-dependent and frequency-independent selection can give rise to three non-trivial equilibria. Not more than two of these will be stable. For example, suppose that $\mathrm{W}_{1}=\mathrm{W}_{3}=\mathrm{I}, \mathrm{W}_{2}=0.8$ and $t=0.3$. Then equation (4) becomes

$$
\mathrm{O} \cdot \mathrm{I}-\mathrm{0} \cdot 9^{8} \hat{q}+2 \cdot 34 \hat{q}^{2}-\mathrm{I} \cdot 5^{6} \hat{q}^{3}=\mathrm{o}
$$

the solutions of which are (approximately) $\hat{q}=0.15, \hat{q}=0.50$ and $\hat{q}=0.85$.

It is possible to test the stability of these equilibria by differentiating the left-hand side of equation (5) and then inserting the values of $\hat{q}$ in the resulting expression. If, when a particular value of $\hat{q}$ is inserted, the sign of the expression is positive, then the equilibrium is unstable. If the sign is negative, the equilibrium is stable (Lewontin, 1958). The logic of this method derives from the fact that the sign of $\Delta q$ is determined by the factor $[b-a-q(2 b-a-c)]$.

The calculation shows that the equilibrium $\hat{q}=0.5$ is unstable, since at that point there is a positive relation between $q$ and $\Delta q$. The other two equilibria are stable. At all three equilibria the heterozygotes are at a selective disadvantage to both homozygotes.

If the heterozygote has a frequency-independent advantage over the homozygotes $\left(W_{1}<W_{2}>W_{3}\right)$, there will be a single equilibrium. Even at this point, if $t$ is large in relation to the differences between $\mathrm{W}_{1}, \mathrm{~W}_{2}$ and $\mathrm{W}_{3}$, the heterozygotes may be at an overall selective disadvantage.

\section{(iv) Combined effects of frequency-dependent and frequency-independent selection with dominance}

If $\mathrm{A}$ is dominant to $\mathrm{A}^{1}$ for characters subject to frequency-dependent selection, the situation can be represented as follows:
Genotypes
AA
$\mathrm{AA}^{1}$
$\mathbf{A}^{1} \mathbf{A}^{1}$
Selective
values

$$
a=\mathrm{W}_{1}\left(\mathrm{I}-t+t q^{2}\right) \quad b=\mathrm{W}_{2}\left(\mathrm{I}-t+t q^{2}\right) \quad c=\mathrm{W}_{3}\left(\mathrm{I}-t q^{2}\right) .
$$


The gene $A^{1}$ will spread from an initially low frequency if $W_{2}>W_{1}$. The gene A will similarly spread if $\mathrm{W}_{2}(\mathrm{I}-2 t p)>\mathrm{W}_{3}(\mathrm{I}-t+2 t p)$. Substituting the values of $a, b$ and $c$ in equation (3), we find that $\hat{q}$ is solved by

$$
\begin{aligned}
\left(\mathrm{W}_{2}-\mathrm{W}_{1}\right)(\mathrm{I}-t)-\hat{q} & \left(2 \mathrm{~W}_{2}-2 t \mathrm{~W}_{2}+t \mathrm{~W}_{1}-\mathrm{W}_{1}-\mathrm{W}_{3}\right) \\
& +t \hat{q}^{2}\left(\mathrm{~W}_{2}-\mathrm{W}_{1}\right)-t \hat{q}^{3}\left(2 \mathrm{~W}_{2}-\mathrm{W}_{1}+\mathrm{W}_{3}\right)=0 .
\end{aligned}
$$

This equation allows three possible solutions, but usually only one is a stable equilibrium. For instance, if $\mathrm{W}_{1}=\mathrm{W}_{3}=\mathrm{I}, \mathrm{W}_{2}=0.8$ and $t=0.3$, then $\hat{q}=0.26$ or $\hat{q}=0.88$. By differentiating the lefthand side of equation (6) and substituting the values of $\hat{q}$, we find that only the latter equilibrium is stable. At this point the heterozygote is at a selective disadvantage to both homozygotes, although this is a rarer occurrence in the presence of dominance. It can never happen, of course, if $\mathrm{W}_{2}$ exceeds $\mathrm{W}_{1}$ and $\mathrm{W}_{3}$. When $\mathrm{W}_{2}=\mathrm{W}_{1}$, (i.e. when $\mathrm{A}$ is dominant for all components of fitness) there is a single stable equilibrium at which

$$
\hat{q}=\sqrt{\frac{\mathrm{W}_{2}(t-\mathrm{I})+\mathrm{W}_{3}}{t\left(\mathrm{~W}_{2}+\mathrm{W}_{3}\right)}} .
$$

\section{THE TIMING OF SELECTION}

So far, we have assumed that both components of selection act simultaneously. In natural conditions, however, different kinds of selection will often operate at different stages of the life-cycle. If frequency-dependent selection acts first, the formulæ given above will still be applicable, but if some component of frequency-independent selection is the first to act, the formulæ may have to be modified. The proportions of the three genotypes may be altered before frequency-dependent selection comes into action. Modification will only be necessary, however, when the preceding selection is strong (say greater than io per cent.).

\section{CONCLUSIONS AND SUMMARY}

We have set up a number of mathematical models to represent situations in which the selective values of genotypes are negatively related to the frequencies of their phenotypes. These models may apply to mimetic and apostatic polymorphisms.

When frequency-dependent selection acts alone, the heterozygote at stable equilibrium may be at a selective disadvantage to both homozygotes. Frequency-dependent selection is able to maintain a balanced polymorphism even in the face of frequency-independent selection against the heterozygote. There may then be more than one stable equilibrium.

These results show that instantaneous estimates of the fitness of genotypes in a polymorphic population may give a misleading picture of the stability (or otherwise) of the polymorphism. 
Acknowledgments.-We are very grateful to Dr A. J. Cain, Dr J. D. Currey, Dr A. Robertson, Professor P. M. Sheppard and Dr M. Williamson for reading and criticising the manuscript. We wish to thank Professor M. M. Swann, F.R.s., and Professor F. W. Rogers Brambell, F.R.s., for the facilities they have provided.

\section{REFERENCES}

CLARKE, B. 1962a. Balanced polymorphism and the diversity of sympatric species. Taxonomy and Geography, ed. D. Nichols. Systematics Association, Oxford.

CLARKE, B. 1962b. Natural selection in mixed populations of two polymorphic snails. Heredity, $I 7,319-345$.

Fisher, R. A. 1930. The Genetical Theory of Natural Selection, Clarendon Press, Oxford. LEWONTIN, R. C. 1958. A general method for investigating the equilibrium of gene-frequency in a population. Genetics, 43, 419-434.

MAYNARD SMITH, J. 1962. Disruptive selection, polymorphism and sympatric speciation. Nature, 195, 60-62.

SHEPPARD, P. M. 1959. The evolution of mimicry; a problem in ecology and genetics. Cold Spring Harb. Symp. Quant. Biol., 24, 13 I-140.

WRIGHT, s. 1948. On the roles of directed and random changes in gene frequency in the genetics of natural populations. Evolution, 2, 279-294. 